\title{
Olanzapine-induced Orthostatic Hypotension
}

\author{
Amlan Kusum Jana ${ }^{1}$, Samir Kumar Praharaj ${ }^{2}$, Nirmalya Roy ${ }^{1}$ \\ ${ }^{1}$ Department of Psychiatry, KPC Medical College and Hospital, Kolkata, West Bengal, ${ }^{2}$ Department of Psychiatry, Kasturba Medical College, \\ Manipal, Karnataka, India
}

\begin{abstract}
Olanzapine is an atypical antipsychotic which is efficacious in the treatment of schizophrenia. The adverse effect profile for olanzapine is benign except for higher rates of metabolic events. Orthostatic hypotension is less commonly reported with olanzapine as compared to first-generation and few atypical antipsychotics. We report a case where olanzapine, in a dose dependent fashion, caused transient postural hypotension.
\end{abstract}

KEY WORDS: Olanzapine; Antipsychotics; Orthostatic hypotension.

\section{INTRODUCTION}

Olanzapine is an atypical antipsychotic with efficacy in schizophrenia slightly higher than others. ${ }^{1)}$ The extrapyramidal adverse effects are much lower than typical antipsychotics, though it has a higher metabolic adverse effect profile. ${ }^{2)}$ Orthostatic hypotension is common with first-generation antipsychotics with rates up to $77 \%$ compared to $15 \%$ with placebo. ${ }^{3)}$ Among atypical antipsychotics, clozapine, risperidone and quetiapine have higher rates of orthostatic hypotension. ${ }^{3)}$ We report a case where olanzapine, in a dose dependent fashion, caused transient postural hypotension.

\section{CASE}

A 22-year-old female was diagnosed with paranoid schizophrenia 16 months back and was started on olanzapine, which was increased up to $15 \mathrm{mg}$ /day over two weeks. She responded well to it, resuming her higher studies as well as a part-time job of a school teacher. However, after two months, she had sudden falls on five occasions over one week, seriously injuring herself with bruises and lacerations. Vitals were checked on all the occasions

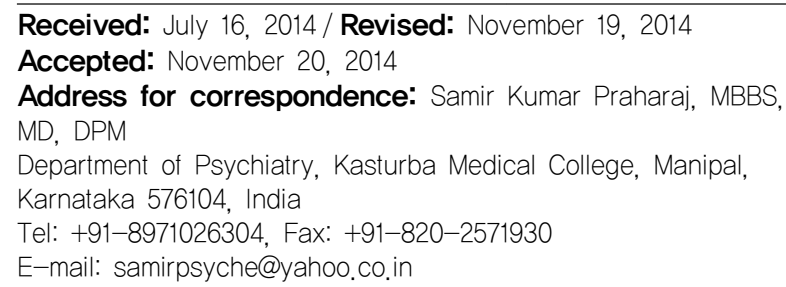

which showed pulse rate ranging from $70-90$ beats per minute (bpm) and blood pressure ranging from 110-130/ $70-80 \mathrm{mmHg}$. However, in all the occasions the examinations could be done after the patient had recovered and gained consciousness. There was no other abnormality on physical examination.

Investigations including complete blood count, fasting and post prandial plasma glucose, lipid profile and liver function test were normal. Her body weight has been maintained at $52 \mathrm{~kg}$ over the past one year, her menstrual cycles were regular and abdominal ultrasonography revealed no abnormalities. Magnetic resonance imaging of brain was also unremarkable. She has a history of childhood asthma and received steroid inhalation eight years back. However, on current assessment both cortisol (9.31 $\mu \mathrm{g} / \mathrm{dl})$ and adrenocorticotropic hormone (14.5 pg/ml) levels were within normal range with respect to age, sex and time (morning/evening) of sampling.

She was advised rest at home, dose of olanzapine was reduced to $10 \mathrm{mg}$ /day and a round-the-clock nurse was appointed for observation and monitoring. Thereafter, the frequency of fall reduced, and there was only one such event when she was dizzy and about to fall but prevented by the attending nurse. Her pulse rate was $140 \mathrm{bpm}$ and blood pressure was $76 / 40 \mathrm{mmHg}$ in supine position, and three minutes later pulse rate was $80 \mathrm{bpm}$ and blood pressure was $110 / 70 \mathrm{mmHg}$ in standing position, which was suggestive of orthostatic hypotension. She recovered from her dizziness and was fully oriented within five minutes of the event. Subsequently, olanzapine was cross-tapered with aripiprazole up to $15 \mathrm{mg}$ /day, on which she is

(c) This is an Open-Access article distributed under the terms of the Creative Commons Attribution Non-Commercial License (http://creativecommons.org/licenses/by-nc/3.0) which permits unrestricted non-commercial use, distribution, and reproduction in any medium, provided the original work is properly cited. 
maintaining well without further recurrence of adverse effects during follow up at two months.

\section{DISCUSSION}

Our patient developed orthostatic hypotension with olanzapine $15 \mathrm{mg}$ per day that subsided following discontinuation of medication. There was no recurrence on administration of aripiprazole. There are few reports of hypotension associated with olanzapine, ${ }^{4,5)}$ one in an elderly individual, and the other with intramuscular administration. The summary of product characteristics for olanzapine reports $5 \%$ subjects develop orthostatic hypotension as compared to $2 \%$ in placebo, ${ }^{6)}$ which is much less as compared to the rates observed with first generation antipsychotics. ${ }^{3)}$ Nevertheless, hypotension and bradycardia has been reported with olanzapine as low as 5 mg per day. ${ }^{7)}$ Most commonly orthostatic hypotension is an incidental finding during routine examinations, and symptomatic hypotension is not usually observed except in the elderly patients. In contrast, our patient, a young female, developed symptomatic orthostatic hypotension with olanzapine that resulted in several episodes of falls, which required treatment discontinuation. Interestingly, Nourian et $a l .{ }^{8)}$ had shown through animal experiments that olanzapine, along with ziprasidone and aripiprazole has the least propensity to cause postural hypotension.

The most accepted mechanism for antipsychotic related hypotension is $\alpha_{1}$-adrenergic blockade. Several other putative mechanisms such as calcium blockade, inhibition of centrally mediated pressor reflexes, and negative inotropic effects, have also been proposed for this adverse effect. ${ }^{9)}$ Sedative effect of olanzapine may additionally contribute to the falls, though such mechanism appeared unlikely in our case. Although the rates of orthostatic hypotension appear lower with olanzapine, an exercise of caution is definitely warranted in the light of the current case.

\section{REFERENCES}

1. Komossa K, Rummel-Kluge C, Hunger H, Schmid F, Schwarz $\mathrm{S}$, Duggan L, et al. Olanzapine versus other atypical antipsychotics for schizophrenia. Cochrane Database Syst Rev 2010;(3):CD006654.

2. Rummel-Kluge C, Komossa K, Schwarz S, Hunger H, Schmid F, Lobos CA, et al. Head-to-head comparisons of metabolic side effects of second generation antipsychotics in the treatment of schizophrenia: a systematic review and meta-analysis. Schizophr Res 2010;123:225-233.

3. Gardener DM, Teehan MD. Antipsychotics and their side effects. New York: Cambridge University Press; 2011.

4. Zacher JL, Roche-Desilets J. Hypotension secondary to the combination of intramuscular olanzapine and intramuscular lorazepam. J Clin Psychiatry 2005;66:1614-1615.

5. Lee TW, Tsai SJ, Hwang JP. Severe cardiovascular side effects of olanzapine in an elderly patient: case report. Int J Psychiatry Med 2003;33:399-401.

6. Lilly. Zyprexa (Olanzapine) Product Monograph. Toronto, Canada:Lilly;2011 [cited 2011 Sep 7]. Available at: http:// pi.lilly.com/us/zyprexa_relprevv.pdf.

7. Markowitz JS, DeVane CL, Boulton DW, Liston HL, Risch SC. Hypotension and bradycardia in a healthy volunteer following a single $5 \mathrm{mg}$ dose of olanzapine. J Clin Pharmacol 2002;42:104-106.

8. Nourian Z, Mow T, Muftic D, Burek S, Pedersen ML, Matz $\mathrm{J}$, et al. Orthostatic hypotensive effect of antipsychotic drugs in Wistar rats by in vivo and in vitro studies of alphal-adrenoceptor function. Psychopharmacology (Berl) 2008;199:15-27.

9. Fayek M, Kingsbury SJ, Zada J, Simpson GM. Cardiac effects of antipsychotic medications. Psychiatr Serv 2001; 52:607-609. 